\title{
Structure-properties relationships of polyhedral oligomeric silsesquioxane (POSS) filled PS nanocomposites
}

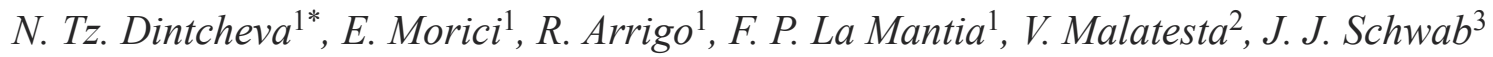 \\ ${ }^{1}$ Dipartimento di Ingegneria Chimica, Gestionale, Informatica, Meccanica, Università di Palermo, Viale delle Scienze, \\ ed.6, 90128 Palermo, Italy \\ ${ }^{2}$ Materials Science Department, Università Milano Bicocca, Via R. Cozzi 53, 20125 Milano, Italy \\ ${ }^{3}$ Hybrid Plastics, 55 WL Runnels Industrial Dr., MS 39401 Hattiesburg, USA
}

Received 23 November 2011; accepted in revised form 20 January 2012

\begin{abstract}
The polyhedral oligomeric silsesquioxane (POSS) additivated polystyrene (PS) based nanocomposites were prepared by melt processing and the structure-properties relationships of the POSS-PS systems were compared to those of the neat PS. In order to investigate the effect of these structural parameters on the final properties of the polymer nanocomposites, five different kinds of POSS samples were used, in particular, POSS with different inorganic cage and with different organic pendent groups. The rheological investigation suggests clearly that the POSS acts as a plasticizer and that the processability of the PS was positively modified. The affinity between the POSS samples and the PS matrix was estimated by the calculated theoretical solubility parameters, considering the Hoy's method and by morphology analysis. Minor difference between the solubility parameter of POSS and the matrix means better compatibility and no aggregation tendency. Furthermore, the POSS loading leads to a decrease of the rigidity, of the glass transition temperature and of the damping factor of the nanocomposite systems. The loading of different POSS molecules with open cage leads to a more pronounced effect on all the investigated properties that the loading of the POSS molecules with closed cage. Moreover, the melt properties are significantly influenced by the type of inorganic framework, by the type of the pendent organic groups and by the interaction between the POSS organic groups and the host matrix, while, the solid state properties appears to be influenced more by the kind of cage.
\end{abstract}

Keywords: nanocomposites, POSS structure, polystyrene matrix, structure-properties relationships

\section{Introduction}

The formulation of high-performance polymer based nanocomposites, e.g. improved stiffness, dimensional stability, barrier properties, fire retardancy, is a very promising and challenging issue from scientific and industrial point of view. The formation of dispersed nanoparticles in polymeric matrices offers unique opportunities for designing advanced structural and functional materials. A small amount of the $1-5 \mathrm{wt} \%$ of nanoparticles in polymer resin gives a significant improvement in properties of the unfilled resin and these improvements are compara- ble to those obtained using $25-40 \mathrm{wt} \%$ microscopic size particles in traditional composite formulations [1-5].

Polyhedral oligosilsesquioxane, so-called POSS, is a class of organosilicic three-dimensional compounds with cage frameworks with different degrees of symmetry and their general formula is $\left(\mathrm{RSiO}_{1.5}\right)_{\mathrm{n}}$, where $n$ is an integer number and $\mathrm{R}$ is an organic group. The organic pendent groups can be designed to achieve the desired affinity with the host polymeric matrix $[1,2]$. Using POSS in polymer based nanocomposites is a promising issue for the design

\footnotetext{
${ }^{*}$ Corresponding author, e-mail: nadka.dintcheva@unipa.it
}

(c) BME-PT 
of materials with high-performance in several applications [6-20] such as low-dielectric applications [6], biomaterials for cardiovascular [14] and dental implants [16], organic solar cells [17], flame resistant nanocomposites [18, 19], optical limiting [20], etc.

An important feature of the POSS formulation is their one-pot preparation, i.e. the cage formation and apex group functionalization occur simultaneously. The formation and functionalization at the same time allows preparation of POSS molecules with controlled and constant structure in contrast to the clay and silica nanoparticles, where the modification is made post-synthesis by ion-exchange reaction in a second step. The easier functionalization of the POSS molecules allows a controlled chemical reaction between the f-POSS and the different functional groups present in the polymer matrix [11, 12]. A recent review addressing the physical properties of POSS polymer based materials and their biomedical applications evidences the needed and desirable characteristics of POSS molecules, i.e. non-toxicity and bio-stability. Bio-stability is indeed, one of the most important consideration for the selection of polymer based composites for medical applications [14].

The POSS loading leads to the improvement of the thermal and mechanical properties comparing to the neat polymer resin $[13,15]$. Furthermore, a few scientific papers report interesting results on the rheology modification of the POSS-additivated polymerbased systems, i.e. the POSS loading decreases the matrix shear viscosity [21, 22]. Moreover, a prolonged thermal treatment of the POSS polymerbased systems, results in migration of POSS molecules to the polymer matrix surface and to the formation of a thin protective POSS rich charred layer [19].

The fundamental understanding of the structureproperties relationships is of utmost importance when developing further applications of the POSS molecules as nanofillers in conventional polymer matrices.

In this paper, the structure-property relationships in POSS filled PS nanocomposites were investigated, the different behaviour of POSS samples with open and closed cage and also with different organic pendent groups were considered. The rheological, mechanical, dynamic-mechanical, thermal and mor- phological analysis of the POSS-PS nanocomposites were evaluated and their properties were compared with those of the neat polystyrene resin. The theoretical calculated solubility parameters of the POSS were compared to the solubility parameter of the PS sample, in order to better understand the nanocomposite properties in molten and solid states. All of the obtained results suggest that the POSS acts as plasticizer. The efficiency of the plasticizing action, investigated in this work, has been found to significantly depend on the kind of the inorganic framework and on the chemical nature and length of the pendent organic groups.

\section{Experimental}

\subsection{Materials}

The materials used in this work are:

- Polystyrene (PS) produced by IneosNova (USA) under the name of 'Empera 251N'; volume melt flow rate $2.4 \mathrm{~cm}^{3} / 10 \mathrm{~min}$ at $200^{\circ} \mathrm{C}$ and $5 \mathrm{~kg}$ load;

- five different polyhedral oligomeric silsesquioxane (POSS) samples, which the structure, molecular weight and chemical formulas are reported in Table $1\left(\right.$ POSS $^{\circledR}$ structures are reported as in Hybrid Plastic Ltd catalogue).

In Table 1, the composition of all the PS based nanocomposites are reported.

\subsection{Preparation and characterization}

The preparation of POSS-PS systems was carried out using a Brabender mixer at $T=170^{\circ} \mathrm{C}$ and mixing speed $50 \mathrm{rpm}$ for 5 minutes. Neat polystyrene was subjected to the same processing.

The rheological characterization, including measurements of the complex viscosity parameters, $G^{\prime}$ and $G^{\prime \prime}$, was performed by using a Rheometric Scientific (USA) RDA II plate-plate rotational rheometer, operating at $T=170^{\circ} \mathrm{C}$ and a $5 \%$ strain deformation. The storage modulus $G^{\prime}$ as a function of the temperature was evaluated in the temperature-sweep modules using strain at $1 \%$ and frequency at $1 \mathrm{~Hz}$. Mechanical tests of rectangular samples cut from compression moulded sheet (sheet thickness about $100 \mu \mathrm{m}$ ) were carried out using a universal Instron machine mod 1122, according to ASTM D882 (crosshead speed of $100 \mathrm{~mm} / \mathrm{min}$ ). The average values of the elastic modulus $(E)$, tensile strength (TS) and elongation at break $(E B)$ were calculated. The reproducibility of the results was about $\pm 5 \%$. 
Table 1. Structure, molecular weight and chemical formula of POSS samples (as reported in the Hybrid Plastics catalogue), sample code and composition of PS based nanocomposites

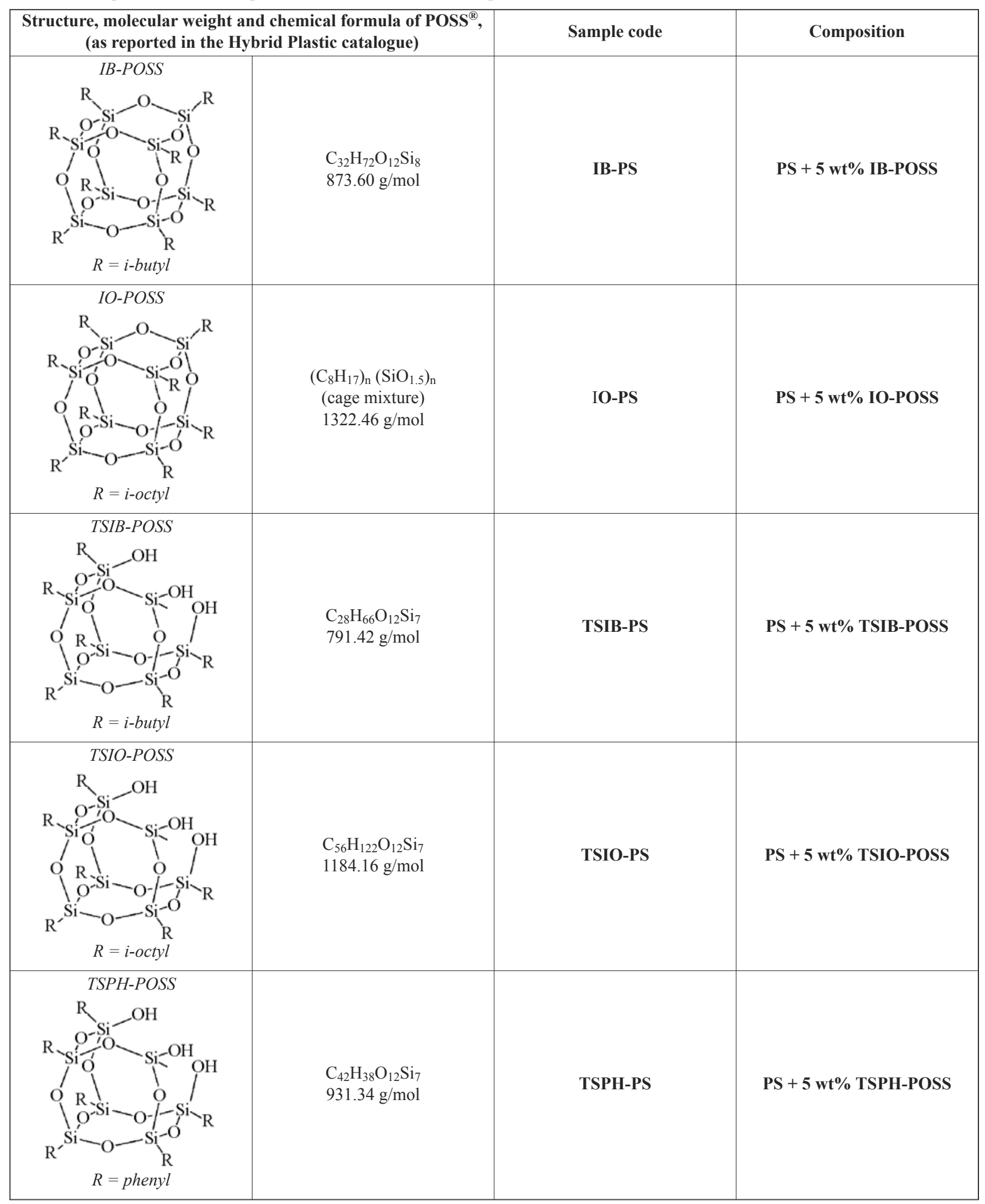

Dynamic Mechanical Thermal Analysis (DMTA) was performed using a Rheometrics DMTA V instrument, single cantilever bending method. The test has been carried out in the temperature swift mode, between 25 and $150^{\circ} \mathrm{C}$ at a heating rate of
$2{ }^{\circ} \mathrm{C} / \mathrm{min}$. The frequency was set to $1 \mathrm{~Hz}$ and the maximum strain amplitude was $0.5 \%$. The storage modulus $\left(E^{\prime}\right)$ and loss factor $(\tan \delta)$ as a function of the temperature were recorded. 
The calorimetric data were evaluated by differential scanning calorimetry (DSC), using a Perkin-Elmer DSC7 calorimeter, at scanning rate of $10^{\circ} \mathrm{C} / \mathrm{min}$.

Scanning Electron Microscopy, SEM, analysis was performed on nitrogen-fractured radial surfaces of all the investigated samples by a Philips (Netherlands) ESEM XL30 equipment.

\section{Results and discussion}

\subsection{Theoretical solubility parameter $\left(\boldsymbol{\delta}_{\text {tot }}\right)$}

The theoretical solubility parameter of the pristine PS and different POSS molecules were calculated considering the Hoy's method [23], as follows:

\begin{tabular}{|l|l|}
\hline $\begin{array}{l}\text { Expressions for } \boldsymbol{\delta} \\
\text { and } \boldsymbol{\delta} \text {-components }\end{array}$ & \multicolumn{1}{|c}{$\mathbf{F}_{\mathrm{i}}+\frac{B}{\bar{n}}$} \\
& $\delta_{\mathrm{tot}}=\frac{V}{}$ \\
& $B=277$ \\
& $\delta_{\mathrm{p}}=\delta_{\mathrm{tot}}\left[\frac{1}{\alpha^{(\mathrm{P})}} \cdot \frac{\mathbf{F}_{\mathrm{p}}}{\mathbf{F}_{\mathrm{t}}+\frac{B}{\bar{n}}}\right]^{1 / 2}$ \\
& $\delta_{\mathrm{h}}=\delta_{\mathrm{tot}}\left[\frac{\alpha^{(\mathrm{P})}-1}{\alpha^{(\mathrm{P})}}\right]^{1 / 2}$ \\
& $\delta_{\mathrm{d}}=\left(\delta_{\mathrm{tot}}^{2}-\delta_{\mathrm{p}}^{2}-\delta_{\mathrm{h}}^{2}\right)^{1 / 2}$ \\
\hline functions & $\mathbf{F}_{\mathrm{t}}=\Sigma N_{\mathrm{i}} \mathbf{F}_{\mathrm{t}, \mathrm{i}}$ \\
& $\mathbf{F}_{\mathrm{p}}=\Sigma N_{\mathrm{i}} \mathbf{F}_{\mathrm{p}, \mathrm{i}}$ \\
& $V=\Sigma N_{\mathrm{i}} V_{\mathrm{i}}$ \\
& $\Delta_{\mathrm{T}}^{(\mathrm{P})}=\Sigma N_{\mathrm{i}} \Delta_{\mathrm{T} T, \mathrm{i}}^{(\mathrm{P})}$ \\
\hline Auxiliary equations & $\alpha^{(\mathrm{P})}=\frac{777 \Delta_{\mathrm{T}}^{(\mathrm{P})}}{V}$ \\
& $n=\frac{0.5}{\Delta_{\mathrm{T}}^{(\mathrm{P})}}$ \\
\hline
\end{tabular}

where:

$\mathbf{F}_{\mathrm{t}}$ is the molar attraction function and $\mathbf{F}_{\mathrm{p}}$ its polar contribution;
$V \quad$ is the molar volume of the solvent molecule or the structural unit of the polymer;

$\Delta_{\mathrm{T}}$ is the Lyndersen correction for non-ideality, $\alpha$ is the molecular aggregation number;

$n \quad$ is the number of repeating units per effective chain segment of the polymer;

$\delta_{\text {tot }}$ is the solubility parameter;

$\delta_{\mathrm{p}}$ is the contribution of the polar forces;

$\delta_{\mathrm{h}}$ is the contribution of the hydrogen bonding;

$\delta_{\mathrm{d}}$ is the contribution of the dispersion forces.

In Table 2, the calculated theoretical solubility parameter values are reported. In general a smaller difference between the solubility parameters of POSS sample and that of the matrix means better compatibility. The theoretical calculated value of the solubility parameter of PS is closer to the values of TSIB-POSS (Table 1), while, the closed cage, IB and IO-POSS have higher calculated solubility parameters. Moreover, the solubility parameter component $\left(\delta_{\mathrm{p}}\right)$ that is related to the contribution of the polar forces and its differences compared to the PS are also reported in the Table 2. In order to ensure better solubility of POSS into the matrix it is necessary to match the polarity of the POSS vertex groups to that of the matrix polymer. Interestingly no solubility parameter component $\left(\delta_{\mathrm{p}}\right)$ related to the contribution of the polar forces for IB and IOPOSS systems was found. Therefore, the type and polarity of the organic pendent group $(\mathrm{R})$ facilitate and control the POSS dispersion into the polymeric matrix. Additionally the organic groups are able to exert a shielding effect of the POSS inorganic framework, i.e. the interaction between the matrix macromolecules and the POSS inorganic core is much less pronounced the longer organic groups. The calculated values of $|\Delta \delta|_{\text {tot }} \mathrm{e}|\Delta \delta|_{\mathrm{p}}$ for TSIBPOSS are minor and considering the length and chemical nature of its pendent organic groups, this POSS samples shows, from a theoretical point of view, the best solubility into the polar polystyrene

Table 2. Values of theoretical calculated solubility parameters of PS and POSS

\begin{tabular}{|c|c|c|c|c|}
\hline Sample & $\begin{array}{c}\delta_{\text {tot }} \\
{\left[\mathrm{J} / \mathrm{cm}^{3}\right]^{1 / 2}(\mathbf{a})}\end{array}$ & $\begin{aligned}|\Delta \delta|_{\text {tot }}= & \left|\delta_{\text {tot PS }}\right|-\left|\delta_{\text {tot POSS }}\right| \\
& {\left[\mathbf{J} / \mathrm{cm}^{3}\right]^{1 / 2} }\end{aligned}$ & $\begin{array}{c}\delta_{\mathbf{p}} \\
{\left[\mathrm{J} / \mathrm{cm}^{3}\right]^{1 / 2}}\end{array}$ & $\begin{aligned}|\Delta \delta|_{\mathrm{p}}= & \left|\boldsymbol{\delta}_{\mathrm{p} \mathrm{PS}}\right|-\left|\boldsymbol{\delta}_{\mathrm{p} \mathrm{POSS}}\right| \\
& {\left[\mathrm{J} / \mathrm{cm}^{3}\right]^{1 / 2} }\end{aligned}$ \\
\hline PS & 19.35 & - & 8.25 & - \\
\hline IB-POSS & 16.05 & 3.30 & 0 & 8.25 \\
\hline IO-POSS & 16.05 & 3.30 & 0 & 8.25 \\
\hline TSIB-POSS & 18.01 & 1.34 & 7.17 & 1.08 \\
\hline TSIO-POSS & 17.12 & 2.23 & 4.80 & 3.45 \\
\hline TSPH-POSS & 21.23 & 1.88 & 12.53 & 4.28 \\
\hline
\end{tabular}

(a)Calculated by Hoy's equation 
matrix and tendency to aggregate less than other POSS samples.

\subsection{Rheological analysis}

In Figures 1a-d, the flow curves and the moduli $G^{\prime}$ and $G^{\prime \prime}$ of pristine PS and POSS additivated PS are reported. The viscosity values in the whole investigated frequency region of all the POSS additivated samples are lower than the those of the pristine polystyrene. In Figures $1 \mathrm{~b}-\mathrm{c}$ the flow curve of closed and open cage POSS additivated PS are shown separately. Similar trends were observed also for the moduli $G^{\prime}$ and $G^{\prime \prime}$, as seen in Figure 1d. It is interesting to highlight that the open cage POSS additivated samples show a slightly pronounced decreases of the viscosity and of the moduli in the whole investigated frequency region, compared to the values of the sample additivated with closed cage POSS. Moreover, the slope of $G^{\prime}$ curve at low frequencies increases by POSS addition and this increase is noticeably higher, for TSIB-PS system. In Table 3, the measured viscosity
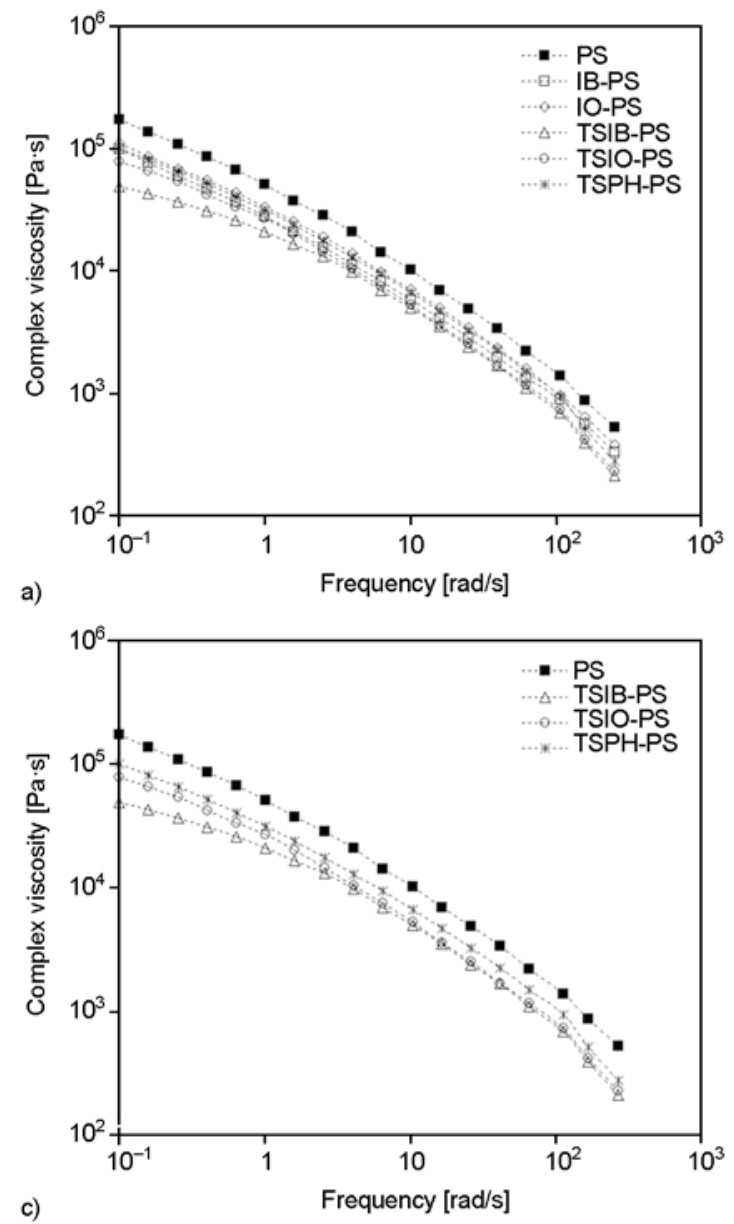

values at low and high frequencies and the values of the cross over frequency are reported. By adding POSS, the interaction between the components (viscosity reduction at low frequency) and easier processability (viscosity reduction at high frequencies) were observed. Moreover, the POSS presence shifts the crossover frequency points between $G^{\prime}$ and $G^{\prime \prime}$ to higher frequencies, as seen in the last column of Table 3. The shift is less pronounced for the systems containing closed-cage POSS. It is interesting to highlight a marked shift of the cross-over point for the TSIB-PS system.

Table 3. Viscosity values at low and high frequencies and cross over between $G^{\prime}$ and $G^{\prime \prime}$ moduli of all the investigated samples

\begin{tabular}{|l|c|c|c|}
\hline \multicolumn{1}{|c|}{ Sample } & $\begin{array}{c}\boldsymbol{\eta}^{*}[\mathbf{P a} \cdot \mathbf{s}] \\
\text { at } \boldsymbol{\omega}=\mathbf{0 . 1} \mathbf{~} \mathbf{r a d} / \mathbf{s}\end{array}$ & $\begin{array}{c}\boldsymbol{\eta}^{*},[\mathbf{P a} \cdot \mathbf{s}] \\
\mathbf{a t} \boldsymbol{\omega} \mathbf{1 0 0} \mathbf{~ r a d} / \mathbf{s}\end{array}$ & $\begin{array}{c}\boldsymbol{\omega}_{\text {cross-over }} \\
{[\mathbf{r a d} / \mathbf{s}]}\end{array}$ \\
\hline PS & $1.72 \cdot 10^{5}$ & $1.39 \cdot 10^{3}$ & 0.25 \\
\hline IB-PS & $0.98 \cdot 10^{5}$ & $0.89 \cdot 10^{3}$ & 0.39 \\
\hline IO-PS & $1.10 \cdot 10^{5}$ & $0.95 \cdot 10^{3}$ & 0.39 \\
\hline TSIB-PS & $0.49 \cdot 10^{5}$ & $0.69 \cdot 10^{3}$ & 1.58 \\
\hline TSIO-PS & $0.77 \cdot 10^{5}$ & $0.73 \cdot 10^{3}$ & 0.63 \\
\hline TSPH-PS & $1.01 \cdot 10^{5}$ & $0.93 \cdot 10^{3}$ & 0.63 \\
\hline
\end{tabular}
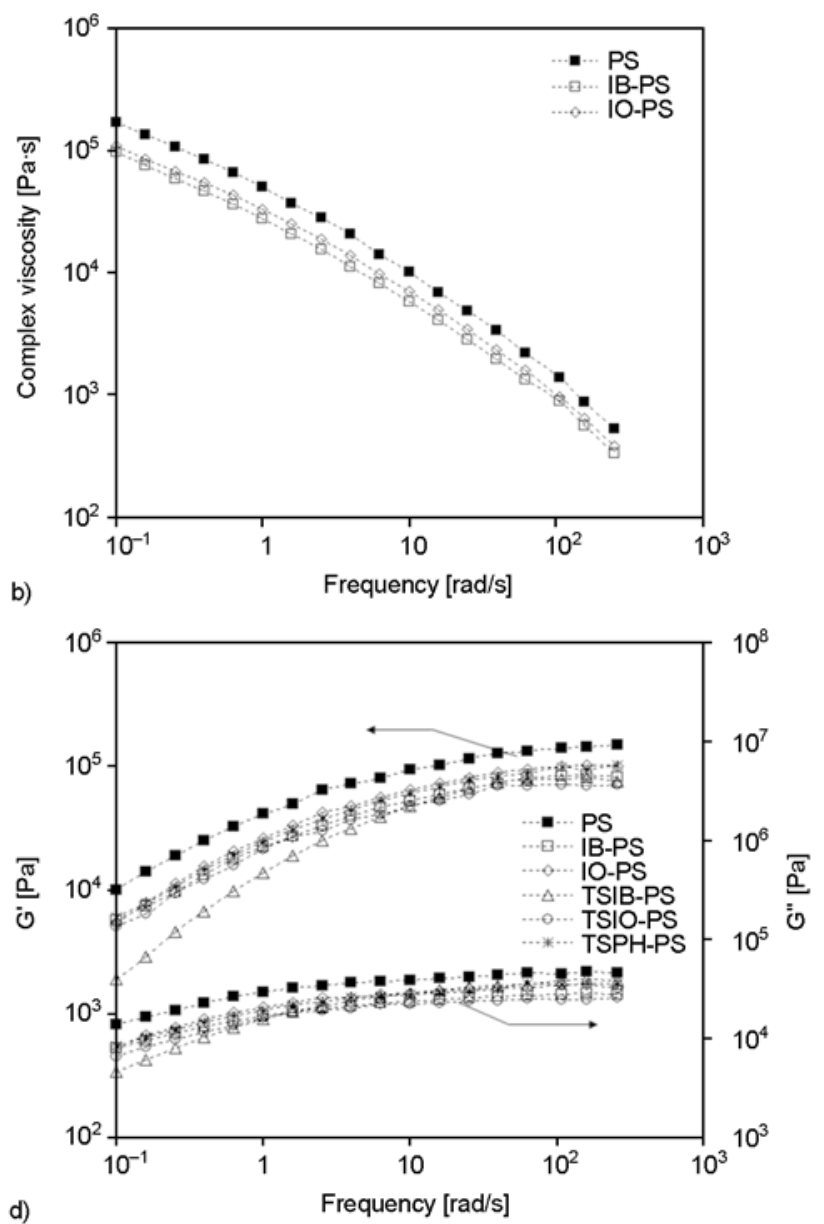

Figure 1. PS and POSS-PS nanocomposites: (a) flow curves of all materials; (b) flow curves of PS additivated with closed cage POSS; (c) flow curves of PS additivated with open cage POSS; (d) $G^{\prime}$ and $G^{\prime \prime}$ moduli of all materials 

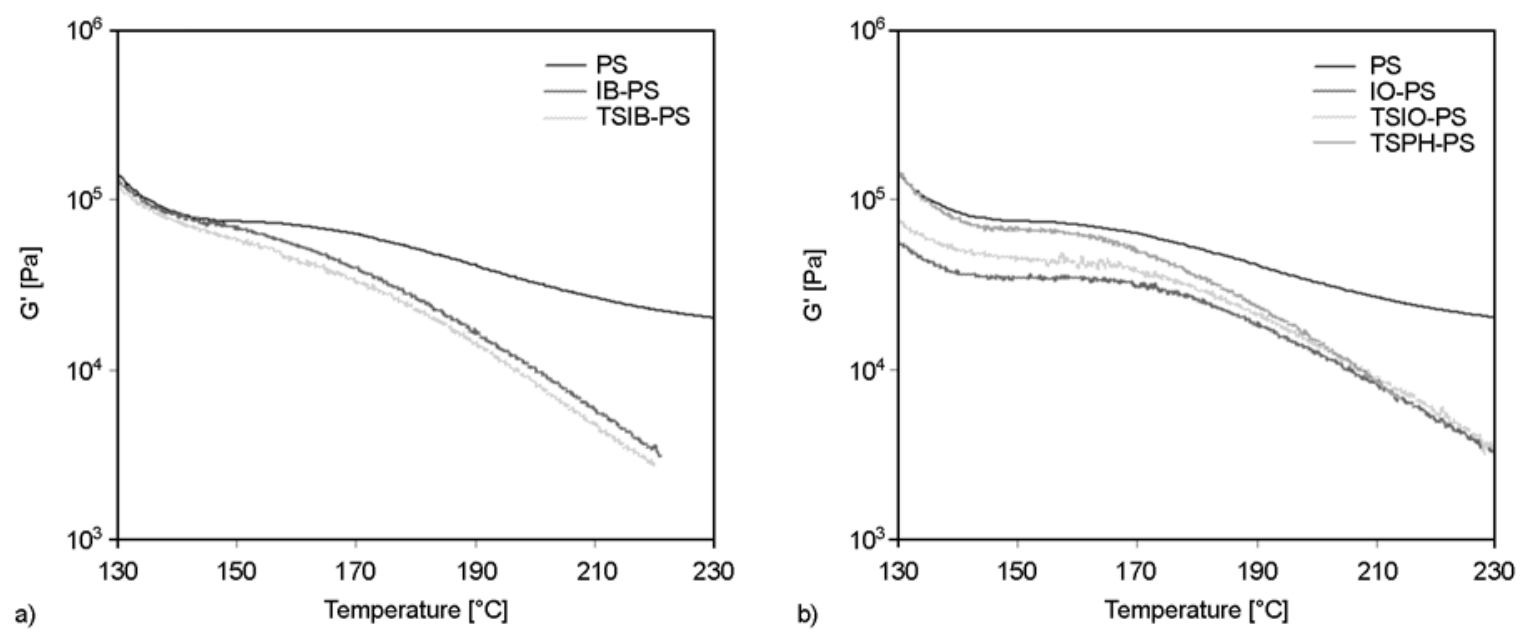

Figure 2. Storage modulus $\left(G^{\prime}\right)$ as a function of the temperature of PS and POSS-PS nanocomposites

Finally, the $G^{\prime}$ modulus was measured as a function of the temperature as seen in Figure 2. The pristine PS shows a clearly pronounced plateau in the $G^{\prime}-T\left({ }^{\circ} \mathrm{C}\right)$ curve in the range between $130-170^{\circ} \mathrm{C}$. The plateau is less extended for all the POSS additivated systems and disappears in the TSIB-PS system. Additionally the slope of the curves at higher temperature values increases significantly in POSS containing PS samples and is highest for TSIB-PS system.

Moreover, all the rheological investigations suggest that the melt properties of the POSS-PS samples are significantly influenced by the POSS presence. In fact, the POSS loading leads to a significant viscosity and moduli decrease and to a less extended plateau (in some case to its disappearance) in the $G^{\prime}$ trend vs temperature curves due to evident a plasticizer and/or lubricant effect, in keeping with what is reported in the literature [22]. The global effect of the POSS loading can be explained considering three synergic actions, i.e. the free volume increase, the reduction of the friction between the macromolecules and the entanglement density decrease due to the penetration and good dispersion of the POSS molecules into matrix. Furthermore, considering the synergic action of these actions, it is possible to suggest that the rheological behaviour of POSS-PS systems is significantly influenced by both inorganic framework and pendent organic groups. The open inorganic cage is more flexible than the close cage and this fact probably favours the reduction of the frictional effect between the matrix macromolecules. The length of the organic pendent groups determines the free volume variations, i.e. the long pendent organic groups favour the increase of the free volume but the short pendent groups make a POSS molecule more mobile and favours the decrease of the entanglement density of the polystyrene macromolecules.

\subsection{Mechanical and dynamic mechanical thermal analysis}

The main mechanical properties, i.e. elastic modulus, $E$, tensile strength, $T S$, and elongation at break, $E B$, were measured and the obtained results are reported in Table 4 . The POSS loading leads to a slight decrease of the elastic modulus and of the tensile strength. In particular, the reduction of both the elastic modulus and tensile strength of the TSIB-PS with respect to the pristine PS is about $8 \%$, while the drop of the TSPH-PS is much less i.e. only about $1.5 \%$. PS is brittle (its elongation at break is about $3 \%$ ) and the POSS loading does not change this value, see Table 4.

In keeping with the tensile test results obtained at room temperature, the POSS loading leads to a decrease of the modulus $E^{\prime}$ at $T=30^{\circ} \mathrm{C}$, obtained by

Table 4. Mechanical properties: elastic modulus, $E$, tensile strength, $T S$ and elongation at break, $E B$ (measured by tensile test) and modulus $E_{30^{\circ} \mathrm{C}}^{\prime}$ (measured by DMTA) of the pristine PS and POSS-PS systems

\begin{tabular}{|l|c|c|c|c|}
\hline \multicolumn{1}{|c|}{ Samples } & $\begin{array}{c}\mathbf{E} \\
{[\mathbf{M P a}]}\end{array}$ & $\begin{array}{c}\text { TS } \\
{[\mathbf{M P a}]}\end{array}$ & $\begin{array}{c}\mathbf{E B} \\
{[\mathbf{\%}]}\end{array}$ & $\begin{array}{c}\mathbf{E}^{\prime}{ }^{\circ}{ }^{\circ} \mathbf{C} \\
{[\mathbf{M P a}]}\end{array}$ \\
\hline PS & $1480 \pm 74.0$ & $34.5 \pm 1.7$ & $3.0 \pm 0.15$ & 1476 \\
\hline IB-PS & $1370 \pm 68.5$ & $32.1 \pm 1.6$ & $3.0 \pm 0.15$ & 1356 \\
\hline IO-PS & $1392 \pm 69.6$ & $31.8 \pm 1.6$ & $3.1 \pm 0.15$ & 1397 \\
\hline TSIB-PS & $1358 \pm 67.9$ & $31.5 \pm 1.5$ & $3.2 \pm 0.16$ & 1329 \\
\hline TSIO-PS & $1362 \pm 68.1$ & $31.5 \pm 1.5$ & $3.0 \pm 0.15$ & 1308 \\
\hline TSPH-PS & $1458 \pm 72.9$ & $34.0 \pm 1.7$ & $3.0 \pm 0.15$ & 1417 \\
\hline
\end{tabular}


DMTA, with respect to the PS, as shown in the last column of Table 4. More information about the trend of the $E^{\prime}$ and the damping factor $(\tan \delta)$ as a function of the temperature can be found in Figure 3. The peak of $\tan \delta$ (values are reported in Table 5, see first column) shifts to a lower temperature by POSS addition and also a slight decrease of the peak intensities was observed. This shift is more pronounced for all the open cage POSS additivated systems and, in particular, for TSIB-PS system.

The decrease of the rigidity of POSS additivated systems may be explained considering the low molecular weight of the pristine POSS systems, see Table 1. Generally, the addition of low molecular weight plasticizers leads to a decrease of the rigidity and in some case, to a rise of the elasticity [24]. The mechanical behaviour of POSS-PS systems seems to be influenced much more by the chemical nature of the pendent groups than the type of the inorganic cage. The elastic modulus values of the POSS-PS systems prepared with open cage POSS are lower than the values of those prepared with

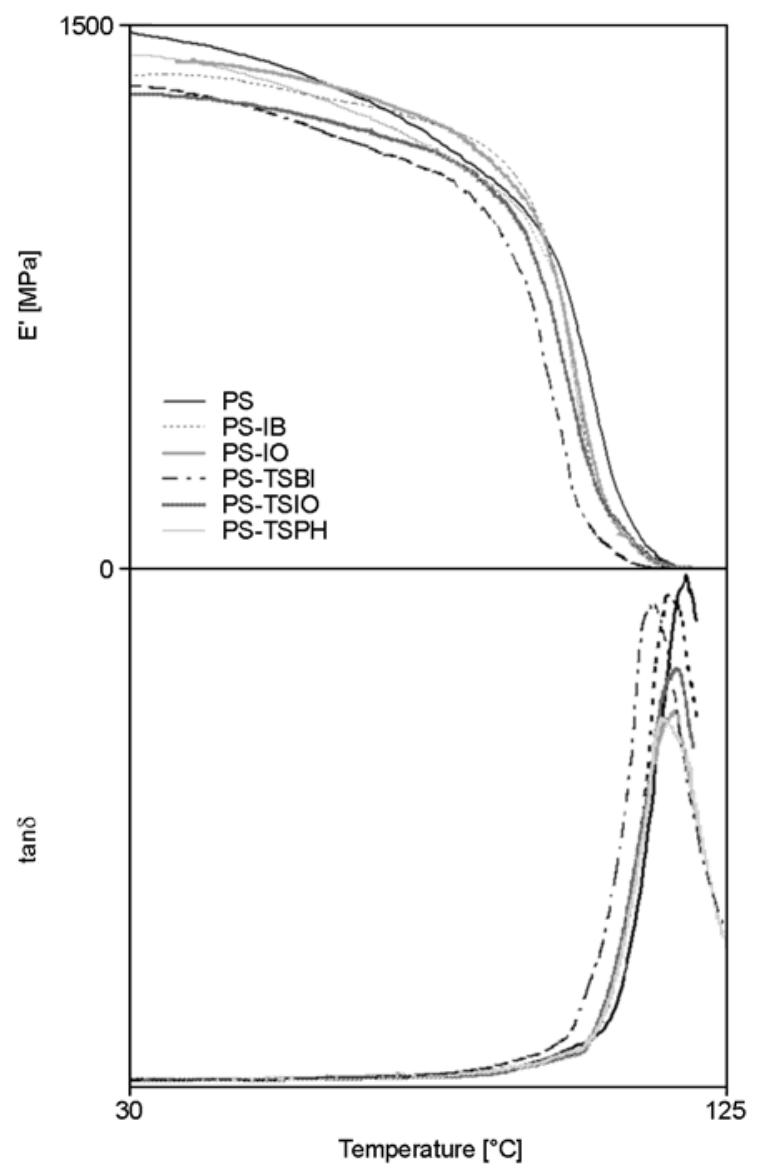

Figure 3. Modulus $\left(E^{\prime}\right)$ and damping factor $(\tan \delta)$ as a function of the temperature of PS and POSS-PS nanocomposites closed cage POSS but at the same time, the solid state properties of TSPH-PS sample are very similar to those of the pristine PS, probably due to the $\pi-\pi$ interaction between the phenyl rings. Furthermore, the change of the macroscopical properties of the POSS based nanocomposites, correlated to a good POSS molecules dispersion, can be obtained considering some chemical interaction between the pendent organic groups and the macromolecules of the host matrix. So, the $\pi-\pi$ interaction may be stronger in PS matrix, taking into account the side position of the benzene ring in polystyrene in comparison with matrices as PKFE [25] or PC [25, 26], in agreement with the literature.

\subsection{Differential scanning calorimetry}

The glass transition temperature, estimated as the mid-point in the heating curves between the onset and end transition points, of neat PS is about $102.0^{\circ} \mathrm{C}$, as reported in Table 5. In Figure 4 is shown the sec-

Table 5. Damping factor (measured by DMTA) and glass transition temperatures (measured by DSC) of pristine PS and POSS-PS systems

\begin{tabular}{|l|c|c|c|c|}
\hline \multirow{2}{*}{ Samples } & \multirow{2}{*}{$\begin{array}{c}\mathbf{T}_{\tan \boldsymbol{\delta}} \\
{\left[{ }^{\circ} \mathbf{C}\right]}\end{array}$} & $\mathbf{T}_{\text {onset }}$ & $\mathbf{T}_{\text {mid-poit }}$ & $\mathbf{T}_{\text {end }}$ \\
\cline { 3 - 5 } & 118.5 & 98.5 & 102.0 & 105.5 \\
\hline PS & 116.8 & 98.0 & 101.3 & 104.0 \\
\hline IO-PS & 116.1 & 95.5 & 100.2 & 105.0 \\
\hline TSIB-PS & 113.5 & 94.2 & 98.0 & 102.5 \\
\hline TSIO-PS & 115.3 & 94.0 & 99.2 & 104.5 \\
\hline TSPH-PS & 115.0 & 95.8 & 99.0 & 104.5 \\
\hline
\end{tabular}

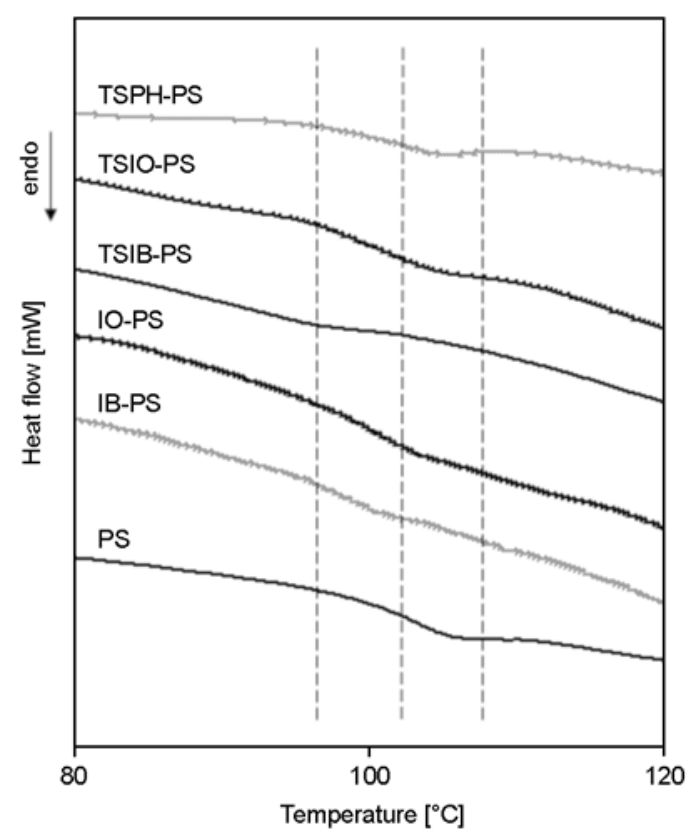

Figure 4. DSC traces of second heating of PS and POSS-PS nanocomposites 

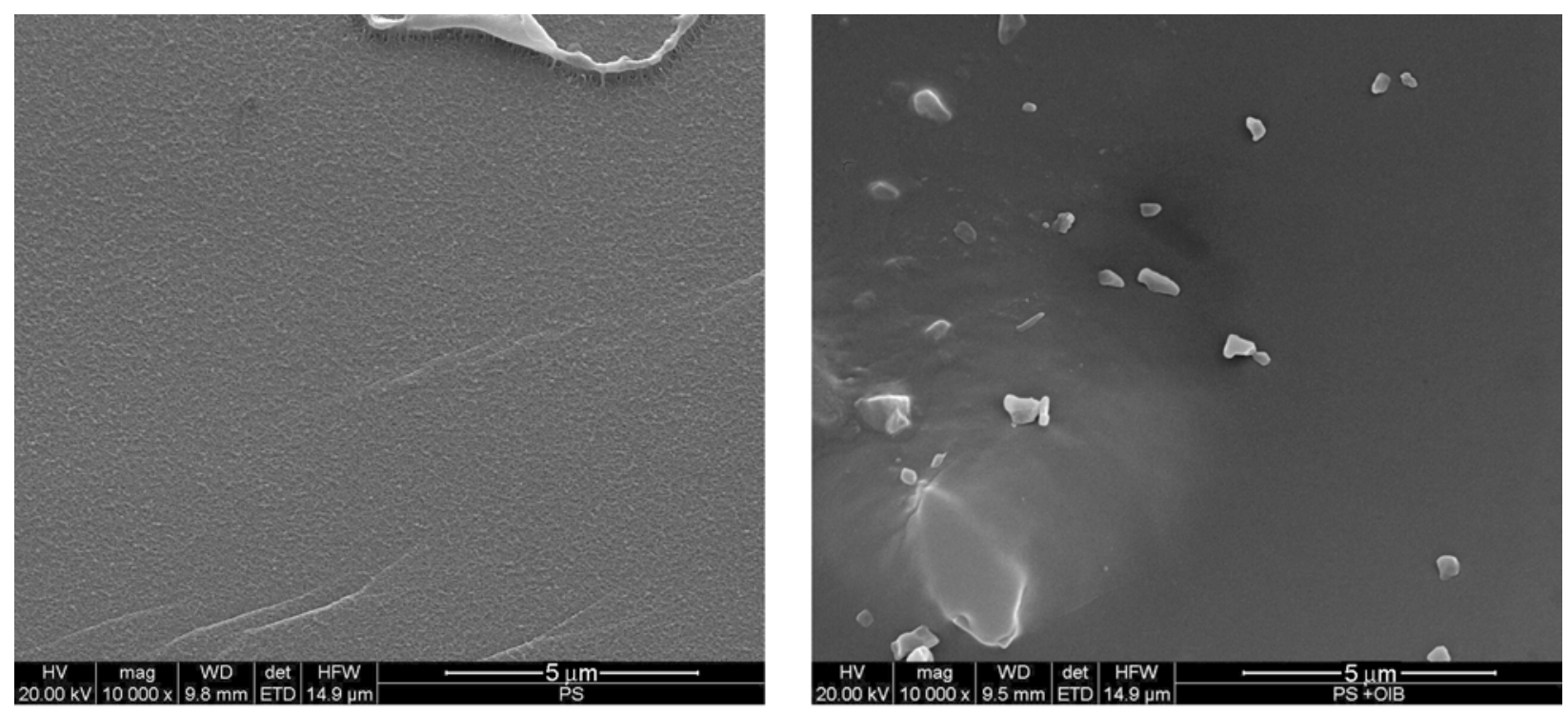

a)
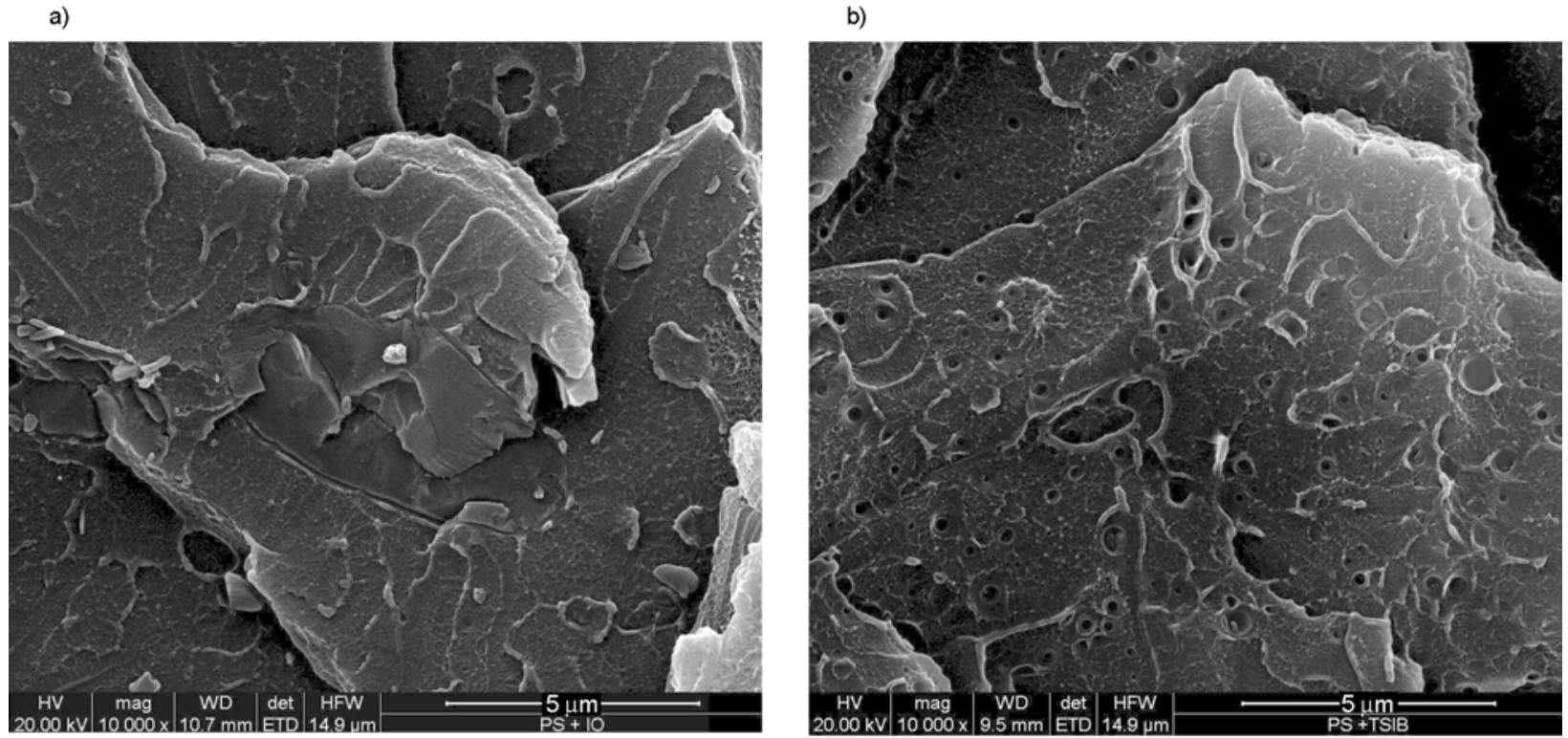

c)

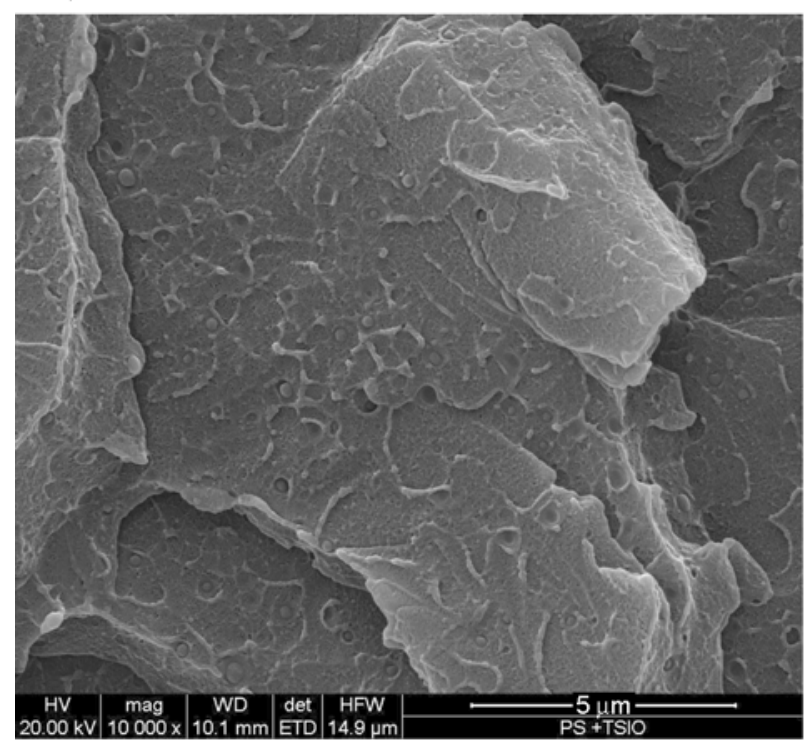

e)

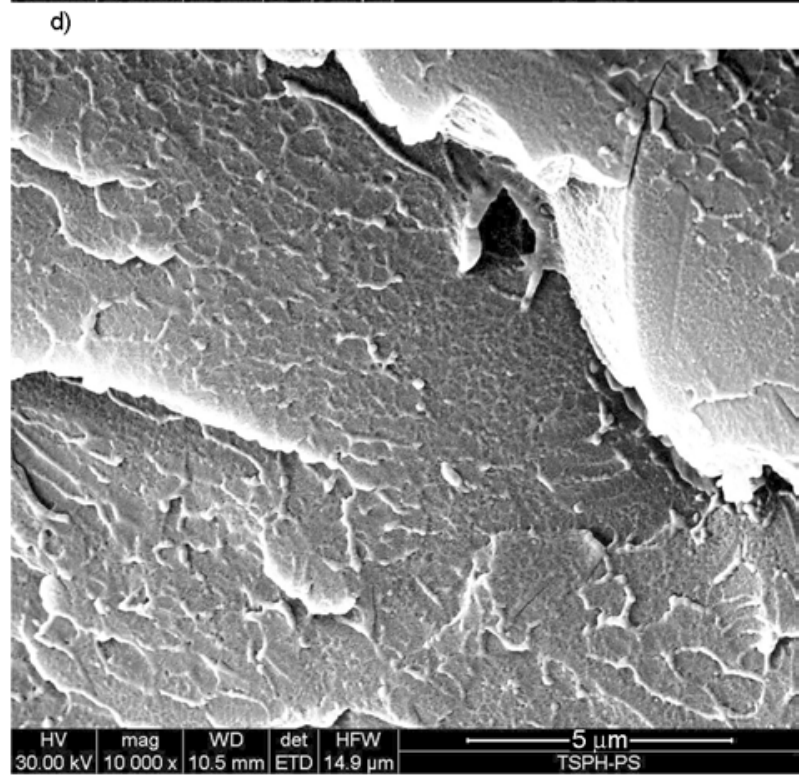

f)

Figure 5. SEM images of all the investigated samples: (a) neat PS; (b) IB-PS; (c) IO-PS; (d) TSIB-PS; (e) TSIO-PS; (f) TSPH-PS 
ond scan of heating scan. The POSS presence leads to a lower glass transition temperature. A similar decrease of the onset, mid-point and end temperatures was noticed. The onset and end temperatures are, respectively, the temperatures at which the transition process starts and finishes for low and high molecular weight PS fractions. A same shifting of the three temperatures to lower temperatures suggests that the nature of the transition of the PS macromolecules is not modified; and only the temperature at which the glass transition occurs is modified due to the POSS presence. In fact, the POSSPS samples exhibit a similar behaviour to that imparted by low molecular weight PS. It is evident that the POSS molecules show a plasticizer effect and this effect is more pronounced for POSS samples with open cage. Moreover, the TSIB-PS sample shows the lowest glass transition, also in keeping with the lowest value of $\tan \delta$. The glass transition temperature seems not to be influenced significantly by the chemical nature of the pendent organic groups, but rather depends on the type of the inorganic framework. In particular, the POSS with open cage induces a more pronounced decrease of the glass transition temperature than the POSS with closed cage.

\subsection{Scanning electron microscopy}

In Figure 5 the SEM image of PS and POSS-PS nanocomposites are shown. The formation of POSS aggregates at micrometric level on the fractured surface of IB-PS sample is clearly noticed (see Figure 5b), while, the POSS-PS nanocomposites show different matrix morphology and also no aggregates can be observed (see Figure 5c-f). The empty holes are visible on the images and they are probably due to the POSS particles attached on the other fractured surfaces. The open cage POSS-PS systems show better dispersion than the closed cage POSSPS nanocomposites. The SEM observations are in total agreement with the calculated theoretical solubility parameters. In particular, the solubility parameter of the IB-POSS is larger than that of the PS and some aggregation between the molecules occurs.

\section{Conclusions}

In this work the structure-properties relationships of the POSS-PS systems were accurately investigated and their variation with respect to that of the neat PS were highlighted. The affinity between the PS matrix and POSS samples was estimated from the theoretical calculated solubility parameters and by morphology analysis. Furthermore, the rheological analysis suggests that the POSS molecule acts as plasticizers in the melt and it is can be explained considering three synergic effects: the increase of the free volume, the reduction of the friction between the PS macromolecules and the decrease of the entanglement density due to the penetration and good dispersion of the POSS molecules into matrix. Additionally the efficiency as plasticizers of the POSS molecules may be related to the structure of their inorganic framework and the chemical nature and length of the pendent organic groups. The open cage POSS system shows a more pronounced plasticizer effect onto PS matrix than the closed cage POSS systems due to the major flexibility of the inorganic framework. The POSS system with closed cage and short organic pendent groups shows a tendency to re-aggregates formation (i.e. IB-POSS), while, the POSS with open cage and same organic groups is the best plasticizer (i.e. TSIB-POSS). The POSS systems with long pendent organic groups favour the increase of the free volume in the nanocomposites but at the same time, do not favour the decrease of the entanglement density (i.e. IO-POSS and TSIO-POSS). It is interesting to highlight that the POSS loading leads to a decrease of the rigidity, glass transition temperature and damping factor. The last effect is less pronounced where the chemical nature of the organic groups is similar to that of the polymeric matrix. In fact, the TSPH-POSS presence does change only slightly the properties of the PS in the solid state, also due to the $\pi$ - $\pi$ interaction between the phenyl rings.

Really, the POSS molecules are expensive additives but considering their performance improvement and non-toxicity could be believe as excellent plasticizers for polymer based nanocomposites in medical and aero-space applications.

\section{Acknowledgements}

This work has been financially supported by University of Palermo, Progetti innovativi ordinari Palermo 2007 'Nuovi materiali compositi nanostrutturati: preparazione e caratterizzazione' (codice OIPA07P92W). 


\section{References}

[1] Harrison P. G.: Silicate cages: Precursors to new materials. Journal of Organometallic Chemistry, 542, 141183 (1997).

DOI: $10.1016 / \mathrm{S} 0022-328 X(96) 06821-0$

[2] Provatas A., Matisons J. G.: Silsesquioxanes: Synthesis and applications. Trends in Polymer Science, 5, 327-332 (1997).

[3] Zheng L., Waddon A. J., Farris R. J., Coughlin E. B.: $\mathrm{X}$-ray characterizations of polyethylene polyhedral oligomeric silsesquioxane copolymers. Macromolecules, 35, 2375-2379 (2002).

DOI: $10.1021 / \mathrm{ma} 011855 \mathrm{e}$

[4] Liu L., Tian M., Zhang W., Zhang L., Mark J. E.: Crystallization and morphology study of polyhedral oligomeric silsesquioxane (POSS)/polysiloxane elastomer composites prepared by melt blending. Polymer, 48, 3201-3212 (2007).

DOI: $10.1016 /$ j.polymer.2007.03.067

[5] Iyer P., Mapkar J. A., Coleman M. R.: A hybrid functional nanomaterial: POSS functionalized carbon nanofiber. Nanotechnology, 20, 325603-325609 (2009). DOI: 10.1088/0957-4484/20/32/325603

[6] Lee Y-J., Huang J-M., Kuo S-W., Lu J-S., Chang F-C.: Polyimide and polyhedral oligomeric silsesquioxane nanocomposites for low-dielectric applications. Polymer, 4, 173-181 (2005).

DOI: $10.1016 /$ j.polymer.2004.10.003

[7] Zhang Y., Lee S., Yoonessi M., Liang K., Pittman C. U.: Phenolic resin-trisilanolphenyl polyhedral oligomeric silsesquioxane (POSS) hybrid nanocomposites: Structure and properties. Polymer, 47, 2984-2996 (2006). DOI: $10.1016 /$ j.polymer.2006.03.005

[8] Hosaka N., Torikai N., Otsuka H., Takahara A.: Structure and dewetting behavior of polyhedral oligomeric silsesquioxane-filled polystyrene thin films. Langmuir, 23, 902-907 (2007).

DOI: $10.1021 / 1 \mathrm{a} 062255 \mathrm{~h}$

[9] Fina A., Bocchini S., Camino G.: Catalytic fire retardant nanocomposites. Polymer Degradation and Stability, 93, 1647-1655 (2008).

DOI: $10.1016 /$ j.polymdegradstab.2008.05.027

[10] Misra R., Alidedeoglu A. H., Jarrett W. L., Morgan S. E.: Molecular miscibility and chain dynamics in POSS/polystyrene blends: Control of POSS preferential dispersion states. Polymer, 50, 2906-2918 (2009). DOI: $10.1016 /$ j.polymer.2009.03.057

[11] Zhang W., Fang B., Walther A., Müller A. H. E.: Synthesis via RAFT polymerization of tadpole-shaped organic/inorganic hybrid poly(acrylic acid) containing polyhedral oligomeric silsesquioxane (POSS) and their self-assembly in water. Macromolecules, 42, 25632569 (2009).

DOI: $10.1021 / \mathrm{ma} 802803 \mathrm{~d}$
[12] Jeon J-H., Lim J-H., Kim K-M.: Hybrid nanocomposites of palladium nanoparticles having POSS and MWNTs via ionic interactions. Macromolecular Research, 17, 987-994 (2009).

DOI: $10.1007 / \mathrm{BF} 03218646$

[13] Tanaka K., Adachi S., Chujo Y.: Structure-property relationship of octa-substituted POSS in thermal and mechanical reinforcements of conventional polymers. Journal of Polymer Science Part A: Polymer Chemistry, 47, 5690-5697 (2009).

DOI: $10.1002 /$ pola. 23612

[14] Wu J., Mather P. T.: POSS polymers: Physical properties and biomaterials applications. Journal of Macromolecular Science Part C: Polymer Reviews, 49, 2563 (2009).

DOI: $10.1080 / 15583720802656237$

[15] Lim S-K., Hong E-P., Choi H. J., Chin I-J.: Polyhedral oligomeric silsesquioxane and polyethylene nanocomposites and their physical characteristics. Journal of Industrial and Engineering Chemistry, 16, 189-192 (2010). DOI: $10.1016 /$ j.jiec.2010.01.049

[16] Mohammad S. A., Wee A. G., Rumsey D. J., Schricker S. R.: Maxillofacial materials reinforced with various concentrations of polyhedral silsesquioxanes. Journal of Dental Biomechanics, 701845/1-701845/6 (2010). DOI: $10.4061 / 2010 / 701845$

[17] Brandhorst H., Isaacs-Smith T., Wells B., Lichtenhan J. D., Fu B. X.: POSS coating as replacements for solar cell cover glasses. in 'Conference Record of the 2006 IEEE $4^{\text {th }}$ World Conference on Photovoltaic Energy Conversion, Waikoloa, USA' 1887-1890 (2007). DOI: 10.1109/WCPEC.2006.279864

[18] Zhang Z., Gu A., Liang G., Ren P., Xie J., Wang X.: Thermo-oxygen degradation mechanisms of POSS/ epoxy nanocomposites. Polymer Degradation and Stability, 92, 1986-1993 (2007).

DOI: 10.1016/j.polymdegradstab.2007.08.004

[19] Tang Y., Lewin M.: Migration and surface modification in polypropylene (PP)/polyhedral oligomeric silsequioxane (POSS) nanocomposites. Polymers for Advanced Technologies, 20, 1-15 (2009).

DOI: $10.1002 /$ pat.1229

[20] Zhang B., Chen Y., Wang J., Blau W. J., Zhuang X., He N.: Multi-walled carbon nanotubes covalently functionalized with polyhedral oligomeric silsesquioxanes for optical limiting. Carbon, 48, 1738-1742 (2010). DOI: $10.1016 /$ j.carbon.2010.01.015

[21] Wu J., Haddad T. S., Kim G-M., Mather P. T.: Rheological behavior of entangled polystyrene-polyhedral oligosilsesquioxane (POSS) copolymers. Macromolecules, 40, 544-554 (2007). DOI: $10.1021 / \mathrm{ma} 061886 \mathrm{f}$ 
[22] Romero-Guzmán M. E., Romo-Uribe A., Zárate-Hernández B. M., Cruz-Silva R.: Viscoelastic properties of POSS-styrene nanocomposite blended with polystyrene. Rheologica Acta, 48, 641-652 (2009). DOI: 10.1007/s00397-009-0358-8

[23] van Krevelen D. W., Nijenhuis K. te: Cohesive properties and solubility. in 'Properties of polymers' (eds.: van Krevelen D. W., Nijenhuis K. te.:) Elsevier, Oxford, 189-228 (2009).

[24] Wypych G.: Handbook of plasticizers. ChemTec Publishing, Toronto (2004).
[25] Iyer S., Schiraldi D. A.: Role of specific interactions and solubility in the reinforcement of bisphenol A polymers with polyhedral oligomeric silsesquioxanes. Macromolecules, 40, 4942-4952 (2007).

DOI: $10.1021 / \mathrm{ma} 0611801$

[26] Sánchez-Soto M., Schiraldi D. A., Illescas S.: Study of the morphology and properties of melt-mixed polycarbonate-POSS nanocomposites. European Polymer Journal, 45, 341-352 (2009).

DOI: $10.1016 /$ j.eurpolymj.2008.10.026 